\title{
Age-Related Pathology Associated with H1N1 A/California/07/2009 Influenza Virus Infection
}

Stephanie J. Bissel, ${ }^{*}$ Chalise E. Carter ${ }^{\dagger}{ }^{\text {Guoji Wang, }}{ }^{*}$ Scott K. Johnson, ${ }^{\dagger}$ Lauren P. Lashua, ${ }^{\ddagger}$ Alyson A. Kelvin, ${ }^{\natural}$ Clayton A. Wiley, ${ }^{*}$ Elodie Ghedin, ${ }^{\ddagger \|}$ and Ted M. Ross ${ }^{\dagger * *}$

From the Department of Pathology, * University of Pittsburgh, Pittsburgh, Pennsylvania; the Center for Vaccines and Immunology, ${ }^{\dagger}$ and the Department of Infectious Diseases, ** University of Georgia, Athens, Georgia; the Center for Genomics \& Systems Biology, ${ }^{\ddagger}$ Department of Biology, College of Arts \& Sciences, New York University, New York, New York; the Department of Microbiology and Immunology, ${ }^{\S}$ Dalhousie University, Halifax, Nova Scotia, Canada; the Canadian Centre for Vaccinology, "Department of Pediatrics, IWK Health Centre, Halifax, Nova Scotia, Canada; and the Department of Epidemiology," College of Global Public Health, New York University, New York, New York

\author{
Accepted for publication \\ August 20, 2019. \\ Address correspondence to \\ Stephanie J. Bissel, Ph.D., Stark \\ Neurosciences Research Insti- \\ tute, Dept of Medical and \\ Molecular Genetics, Indiana \\ University School of Medicine, \\ $320 \mathrm{~W} 15$ th St., NB 214E, \\ Indianapolis, IN 46202- \\ 2266. E-mail: sbissel@iu.edu.
}

Influenza virus infection causes a spectrum of diseases, ranging from mild upper respiratory tract infection to severe lower respiratory tract infection, that can lead to diffuse alveolar damage, interstitial and airspace inflammation, or acute respiratory failure. Mechanisms instructing disease severity are not completely understood, but host, viral, and bacterial factors influence disease outcome. With age being one host factor associated with a higher risk of severe influenza, we investigated regional pulmonary distribution and severity of pneumonia after 2009 H1N1 influenza virus infection in newly weaned, adult, and aged ferrets to better understand age-dependent susceptibility and pathology. Aged ferrets exhibited greater weight loss and higher rates of mortality than adult ferrets, whereas most newly weaned ferrets did not lose weight but had a lack of weight gain. Newly weaned ferrets exhibited minimal pneumonia, whereas adult and aged ferrets had a spectrum of pneumonia severity. Influenza virus-induced pneumonia peaked earliest in adult ferrets, whereas aged ferrets had delayed presentation. Bronchial severity differed among groups, but bronchial pathology was comparable among all cohorts. Alveolar infection was strikingly different among groups. Newly weaned ferrets had little alveolar cell infection. Adult and aged ferrets had alveolar infection, but aged ferrets were unable to clear infection. These different age-related pneumonia and infection patterns suggest therapeutic strategies to treat influenza should be tailored contingent on age. (Am J Pathol 2019, 189: 2389-2399; https://doi.org/10.1016/j.ajpath.2019.08.017)
Infants, young children, and individuals 65 years and older are more susceptible to developing severe disease caused by influenza A virus infection. ${ }^{1-3}$ The developing lungs of children under 5 are vulnerable to obstruction of airflow and surfactant dysfunction induced by respiratory infection, resulting in approximately 20,000 flu-related hospitalizations in the United States since $2010 .^{2}$ In elderly populations, influenza virus infections are among the leading causes of morbidity and mortality, with an estimated $71 \%$ to $85 \%$ of seasonal influenza-related deaths occurring in elderly people. ${ }^{3,4}$ This increased susceptibility to infectious diseases is thought to arise from less effective immune responses. Age-related changes include curtailed antibody production, qualitative antibody changes, diminished B-cell activation, diminished number of naïve $\mathrm{T}$ cells and impairment of their induction, and contraction of the T-cell repertoire. $^{5-8}$ An increase in the basal levels of many inflammatory cytokines, such as tumor necrosis factor (TNF)- $\alpha$, interferon (IFN)- $\gamma$, IL-1 $\beta$, and IL-6, is noted in the aging population. ${ }^{9}$ During influenza virus infection, aged

\footnotetext{
Supported by NIH grant U01AI111598 (E.G., A.A.K., S.J.B., C.A.W., and T.M.R.) and the Georgia Research Alliance (T.M.R.).

Disclosures: None declared.

Current address of S.J.B., Stark Neurosciences Research Institute, Department of Medical and Molecular Genetics, Indiana University School of Medicine, Indianapolis, IN.
} 
individuals have an impaired memory T-cell response to conserved epitopes in influenza viral proteins. ${ }^{10}$

In contrast to seasonal influenza, pandemic outbreaks caused by transmission of a novel influenza virus subtype or strain can exhibit different age-dependent patterns of infection in a population. For example, in 2009, older children and adults between the ages of 5 and 59 years were disproportionately affected during the $\mathrm{H} 1 \mathrm{~N} 1 \mathrm{pdm} 09$ virus pandemic. ${ }^{11}$ Severe cases of H1N1pdm09 infection with A/California/07/ 2009 (CA/09)-like viruses were more frequent in these age groups and were associated with inflammation, leukocyte infiltration, and impaired gas exchange attributable to H1N1pdm-induced lung damage. Healthy infants had a lower proportion of deaths than observed in the prior seasonal influenza seasons. ${ }^{12-14}$ Pediatric patients infected with H1N1pdm09 also experienced mild to moderate clinical disease. ${ }^{15-17}$ Individuals $\geq 70$ years of age had more diverse circulating antibody profiles against the internal genes and the HA1 receptor binding site as well as higher antibody binding affinity to the HA1 head of the CA/09-like viruses compared with antisera from younger individuals. ${ }^{18}$ It is likely that this diverse antibody repertoire reflected exposures to previous H1N1-like viruses, including the 1918-like viruses, swineorigin H1N1 virus from 1976, and/or vaccinations against seasonal strains. ${ }^{19-22}$ A similar outcome is observed in ferrets that were experimentally infected with multiple seasonal-like H1N1 viruses that represented different historical eras and elicited an antibody profile that bound and neutralized H1N1pdm09-like viruses. ${ }^{23}$

To better understand age-dependent susceptibility and pathology of CA/09-like viral infections and severity of pneumonia, a H1N1pdm09 virus (A/California/07/2009) was used to infect newly weaned (6 to 7 weeks of age), adult (6 to 12 months of age) and aged ferrets (5.5 to 7 years of age). The ferrets were observed for up to 14 days post infection (DPI). Ferrets are naturally susceptible to human influenza viruses and recapitulate clinical symptoms, viral pathogenesis, immune responses, and lung development in a similar manner as humans. ${ }^{24,25}$ All age groups had subsets of animals with mild, moderate, and severe morbidity. However, $>50 \%$ of aged ferrets had more severe disease with similar immune dysfunctions that are observed in elderly individuals. Although newly weaned ferrets were more resistant to disease and efficiently cleared viral infection, these animals did not follow normal growth and weight gain patterns as observed in noninfected ferrets.

\section{Materials and Methods}

\section{Influenza Virus and Infection of Ferrets}

Female Fitch ferrets (Mustela putorius furo) were obtained from Triple F Farms (Sayre, PA) and were seronegative to circulating influenza $\mathrm{A}(\mathrm{H} 1 \mathrm{~N} 1$ and $\mathrm{H} 3 \mathrm{~N} 2)$ and $\mathrm{B}$ viruses. Newly weaned ferrets were defined as 6 to 7 weeks of age. Adult ferrets were defined as 6 to 12 months of age. Aged ferrets were defined as 5.5 to 7 years of age. Ferrets were pair housed in stainless steel cages (Shor-Line, Kansas City, KS) that contained Sani-Chips laboratory animal bedding (P.J. Murphy Forest Products, Montville, NJ) and provided with food and fresh water ad libitum. To determine the inoculum dose for each age group, various doses were evaluated with the goal of achieving a mean of $20 \%$ weight reduction no earlier than 8 DPI. To achieve a mean of $20 \%$ weight loss no earlier than 8 DPI, adult and newly weaned ferrets were infected intranasally with H1N1pdm09 virus A/California/07/2009 [Influenza Reagents Resource (IRR), BEI Resources, the Centers for Disease Control and Prevention, Manassas, VA] at a dose of $10^{6}$ plaque-forming units (PFU), whereas a dose of $10^{5}$ PFU was used for aged ferrets. The animals were monitored daily for severity of clinical disease using weight loss. Disease symptoms, including elevated temperature, low activity level, sneezing, and nasal discharge, were noted if present (data not shown). Any animal reaching $>20 \%$ weight loss was humanely euthanized. Ferrets were randomly assigned to be removed from the study at $1,3,5,8$, or 14 DPI unless their clinical conditions (eg, loss of $>20 \%$ body weight) required a humane end point. Blood was collected from anesthetized ferrets via the anterior vena cava after infection. Serum was harvested and frozen at a means $\pm \mathrm{SD}$ of $-20^{\circ} \mathrm{C} \pm 5^{\circ} \mathrm{C}$. The University of Georgia Institutional Animal Care and Use Committee approved all experiments, which were conducted in accordance with the NIH's Guide for the Care and Use of Laboratory Animals, ${ }^{26}$ The Animal Welfare Act, and the Biosafety in Microbiological and Biomedical Laboratories guide of the Centers for Disease Control and Prevention and the NIH.

\section{Tissue}

After serum was collected, necropsies were performed to collect lung tissue. Lungs were rinsed with cold phosphatebuffered saline via catheterized trachea to collect bronchoalveolar lavage fluid for another study. The right upper and lower lobes were removed, and each lobe was sectioned into quadrants. Sections were snap frozen. The left upper and lower lung lobes were formalin perfused. After fixation, tissue was paraffin embedded and 5 - $\mu \mathrm{m}$-thick sections were prepared for histopathologic analysis.

\section{Viral Plaque Assay}

Plaque assays were performed to determine viral burden in nasal washes and lung tissue. ${ }^{27,28}$ Nasal washes were collected after infection, snap frozen, then stored at $-80^{\circ} \mathrm{C}$ until use. Lung tissue supernatants were obtained from frozen lung pieces that were gently thawed on ice, forced through a cell strainer $(70 \mu \mathrm{m})$ and syringe plunger in phosphate-buffered saline, then spun down $(1342 \times g$, 5 minutes, $4^{\circ} \mathrm{C}$ ) to collect supernatant. Nasal washes and lung supernatants were diluted in Iscove's modified Dulbecco's minimum essential medium. 
Madin-Darby canine kidney cells were plated $\left(5 \times 10^{5}\right)$ in each well of a six-well plate. Samples were diluted (final dilution factors of $10^{0}$ to $10^{-6}$ ) and overlaid onto the cells in $100 \mu \mathrm{L}$ of Dulbecco's modified Eagle's medium supplemented with penicillin-streptomycin and incubated for 1 hour. Samples were removed, cells were washed twice, and medium was replaced with $2 \mathrm{~mL}$ of $\mathrm{L} 15$ medium plus $0.8 \%$ agarose (Cambrex, East Rutherford, $\mathrm{NJ}$ ) and incubated for 72 hours at $37^{\circ} \mathrm{C}$ with $5 \%$ carbon dioxide. Agarose was removed and discarded. The cells were fixed with $10 \%$ buffered formalin and then stained with $1 \%$ crystal violet for 15 minutes. After thorough washing in distilled water to remove excess crystal violet, the plates were dried, number of plaques was counted, and the number of PFU per milliliter was calculated.

\section{Histopathologic Analysis}

Tissue sections that contained trachea, lobe from the left upper lung, and lobe from the left lower lung were stained with hematoxylin and eosin. Sections were scored by two blinded readers (S.J.B. and C.A.W.) for percentage of lung involvement and bronchial and alveolar severity. Percentage of lung involvement was defined as the percentage of lung that had histologic pneumonia and was scored as follows: 0 , $\leq 10 \% ; 1,10 \%$ to $25 \% ; 2,26 \%$ to $50 \%$; and $3, \geq 50 \%$. Regional severity of bronchial (bronchi and bronchioles) and alveolar spaces was assessed by the following scoring guidelines: 0 , normal; 1 , mild pneumonia; 2, moderate pneumonia; and 3 , severe pneumonia. ${ }^{21,29}$

\section{In Situ Hybridization}

Sense and antisense templates were generated from a 259bp segment of influenza A virus matrix protein. ${ }^{30}$ S-labeled riboprobes were synthesized using a Riboprobe in vitro transcription system (Promega, Madison, WI). Hybridization was performed on deparaffinized formalin-fixed, paraffin-embedded tissue sections of lung and trachea, as described previously. ${ }^{31}$ The influenza riboprobe had no hybridization to noninfected tissue. The severity of influenza infection in trachea, bronchi and bronchioles, alveolar spaces, and submucosal glands was determined by scoring of influenza virus in situ hybridization (ISH) foci: 0, no definitive signal; 1 , occasional focus; 2 , focus in most fields; and $3,>1$ focus per field. ${ }^{21,29}$

\section{Pneumonia and Influenza Virus Infection Composite Scores}

Pneumonia was evaluated by determination of the percentage of lung involved and bronchial and alveolar severity, whereas influenza infection severity was evaluated in the trachea, bronchiolar, alveolar spaces, and submucosal glands compartments as described above. Composite scores were derived by adding the scores for each lobe (upper and lower lobes) and each compartment. Pneumonia composite scores were scores for percentage of lung involvement. bronchial severity, and alveolar severity. Infection severity scores were scores for bronchial, alveolar, tracheal, submucosal infection.

\section{Statistical Analysis}

Differences in weight loss, sickness score, and viral titers were analyzed by two-way analysis of variance followed by Bonferroni's posttest for each group at multiple time points. Statistical significance was defined as $P<0.05$. Statistical analyses were performed using GraphPad Prism software version 7.0a (GraphPad Software, San Diego, CA).

\section{Data Availability}

The data set pertaining to this study can be directly accessed at Synapse (https://www.synapse.org//\#!Synapse:syn18421089). In addition, this work is part of an integrated data set that is openly accessible for further analysis at Synpase (https://www. synapse.org//\#! Synapse:syn2395480/wiki/63122, both Synapse data sets were last accessed March 27, 2019). Data and metadata, including transcriptomics, viral genomics, microbiome, physiologic, virologic, immunologic, and outcome data, are available for each ferret.

\section{Results}

\section{Aged Ferrets Exhibit Greater Weight Loss and Higher Rates of Mortality}

Newly weaned, adult, and aged female ferrets were infected intranasally with $\mathrm{CA} / 09\left(10^{5}\right.$ to $\left.10^{6} \mathrm{PFU}\right)$ and monitored daily for clinical illness and weight loss. Using weight loss to define severity of disease, ferrets were classified as having mild, moderate, or severe disease and morbidity associated with infection. Adult ferrets lost between 5\% and $15 \%$ of original body weight by 8 DPI before beginning to recover (Figure 1A). Adult ferrets categorized as severe $(n=18)$ had $>12 \%$ weight loss $(12 \%$ to $20 \%)$, with a mean weight loss of $15 \%$. Adult ferrets with moderate weight loss $(n=28)$ had a mean loss of approximately $10 \%$ of original body weight, whereas adult ferrets with mild disease $(n=16)$ lost weight at a slower rate, with peak weight loss at $5 \%$ to $8 \%$ of original body weight at 6 to 8 DPI.

Aged ferrets had greater weight loss (Figure 1B). Aged ferrets categorized as severe $(n=33)$ had $20 \%$ weight loss and had to be humanely sacrificed, with eight of the aged ferrets reaching clinical end points at 6 to 8 DPI. Aged ferrets in the moderate category $(n=15)$ lost $5 \%$ to $8 \%$ of their original weight, and aged ferrets categorized as mild ( $n=15)$ lost $<1 \%$ to $2 \%$ weight and had weight loss that was statistically similar to noninfected aged ferrets $(P<0.0001)$.

In contrast, newly weaned ferrets had a completely different pattern of weight loss and disease. All the newly 
weaned ferrets appeared healthy and did not have signs of sneezing or lethargy. However, because these animals were actively growing and maturing, they were analyzed for lack of weight gain (Figure 1C). Noninfected, newly weaned ferrets $(n=8)$ increased their body weight by $30 \%$ during the 8 days of observation. Newly weaned animals categorized as severe $(n=9)$ lost a mean of $20 \%$ of their body weight by 6 DPI. Newly weaned ferrets that were categorized as moderate $(n=40)$ lost approximately $5 \%$ of their original body weight, and mild newly weaned ferrets $(n=20)$ had little weight loss or gained approximately $5 \%$ weight.

\section{Influenza Virus Persists Longer in Nasal Washes of Aged Ferrets}

Weight loss during CA/09-like virus infection is often correlated with viral titers in ferret nasal washes after infection. ${ }^{32,33}$ Adult ferrets had a peak in nasal wash viral titers on 3 DPI at $1 \times 10^{5} \mathrm{PFU} / \mathrm{mL}$, regardless of the severity of weight loss (Figure 1E). In contrast, the aged ferrets in the severe category had a mean viral titer in their nasal wash at $1 \times 10^{6}$ $\mathrm{PFU} / \mathrm{mL}$, which was 1 to $2 \operatorname{logs}$ higher than ferrets in the mild and moderate category (Figure $1 \mathrm{~F}$ ). Unlike the adult ferrets, virus was detected in the nasal wash of aged ferrets at 1 DPI. The viral titer peaked at 3 DPI, but the virus persisted longer in aged ferrets compared with adult ferrets. Aged ferrets in the moderate and severe category still had detectable virus in their nasal washes at 8 DPI (Figure 1F). Newly weaned ferrets also had detectable viral titers in their nasal washes 1 DPI, and the titers were sustained at $1 \times 10^{5}$ to $1 \times 10^{6} \mathrm{pfu} / \mathrm{mL}$ between 3 and 5 DPI (Figure 1G). These titers peaked at 5 DPI before declining but did not reach baseline by 8 DPI.
Overall, the mean viral titers (Figure $1 \mathrm{H}$ ) did not correlate with pneumonia severity or disease outcome. The mean weight loss (Figure 1D) for each group followed the same trend as pneumonia severity.

\section{Newly Weaned Ferrets Show Mild Pneumonia and Aged Ferrets Exhibit Delayed Presentation}

Evaluation of pneumonia was determined by assessing how much of the lung had pneumonia (percentage of lung involvement) and scoring regional lung compartment pneumonia severity in sets of ferrets that were sacrificed at 1, 3, 5, 8, and 14 DPI (Figure 2). The percentage of lung involvement had different kinetics in each cohort (Figures 2 and 3). The newly weaned ferrets had the least severe lung involvement, peaking between 5 and 8 DPI (Figure 3, A and D). The percentage of lung involvement in adult ferrets peaked at 5 DPI and then began to resolve (Figure 3, A and D). Both newly weaned and adult ferrets continued to present signs of pneumonia at 14 DPI. The aged ferrets had delayed development of pneumonia signs that was significantly different from adult ferrets at $3(P=0.002$ for lower lobe), 5 ( $P=0.03$ for upper lobe and $P<0.0001$ for lower lobe), and 8 ( $P=0.04$ for upper lobe) DPI, and the peak percentage of aged ferret lung involvement was observed at the time requiring euthanasia (8 DPI) (Figure 3, A and D).

\section{Bronchial Pneumonia Is a Feature Common to All Age Groups}

Bronchial pneumonia severity was comparable among all cohorts, but the kinetics varied among the cohorts. Peak severity of bronchial pneumonia ranged from 3 to 8 DPI for newly weaned ferrets and 5 DPI for adults (Figure 3, B and
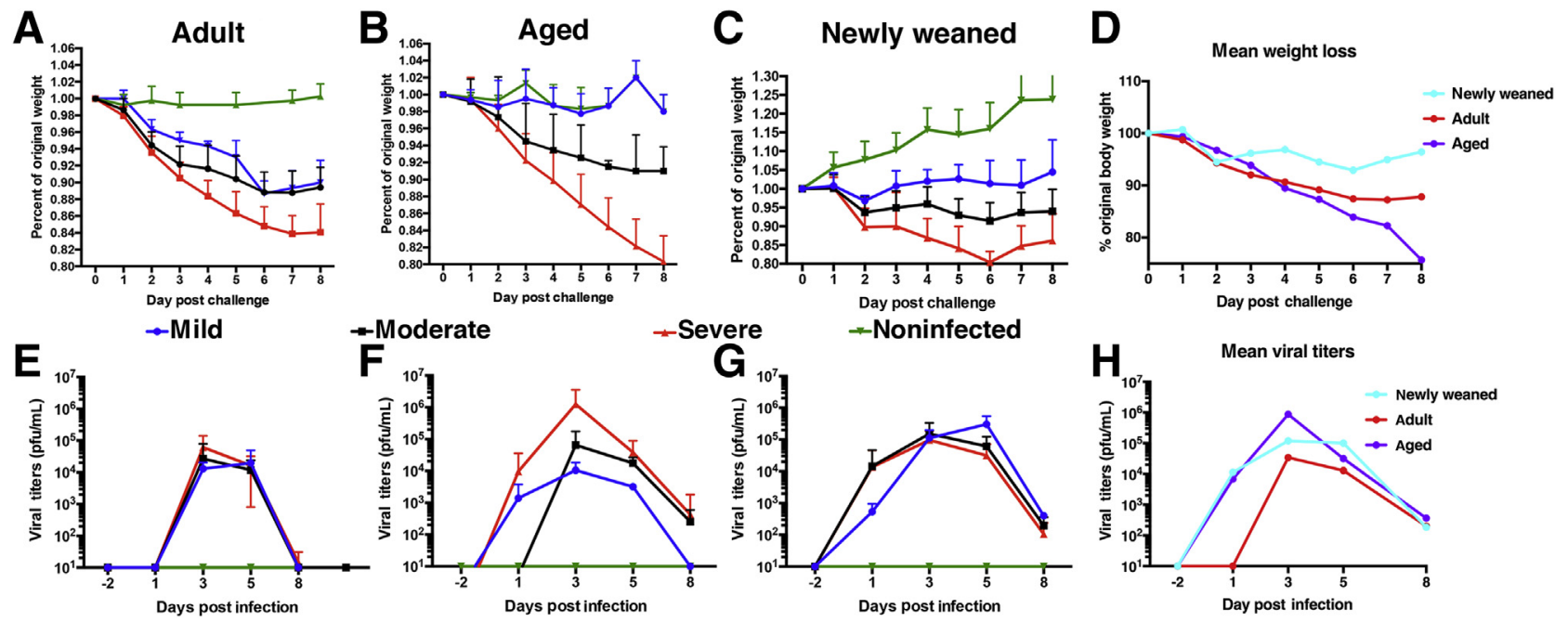

Figure 1 Weight loss and viral nasal wash titers after H1N1pdm09 virus infection. Naïve ferrets were infected with H1N1pdm09 virus (A/California/07/ 2009) and monitored for 2 weeks. A-C: Adult (A), aged (B), and newly weaned (C) ferrets were evaluated daily for weight loss. D: Mean weight loss for each group. E-G: Viral titers were determined from nasal washes collected at 1, 3, 5, and 8 days post infection. H: Mean viral titers for each group. Data are expressed as means \pm SD virus titers. 

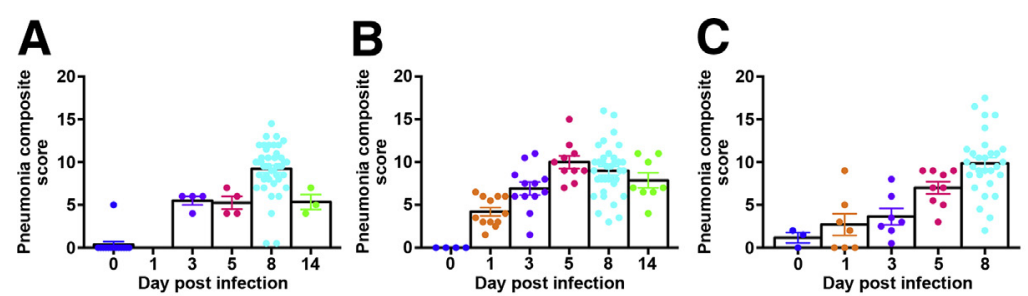

Newly weaned
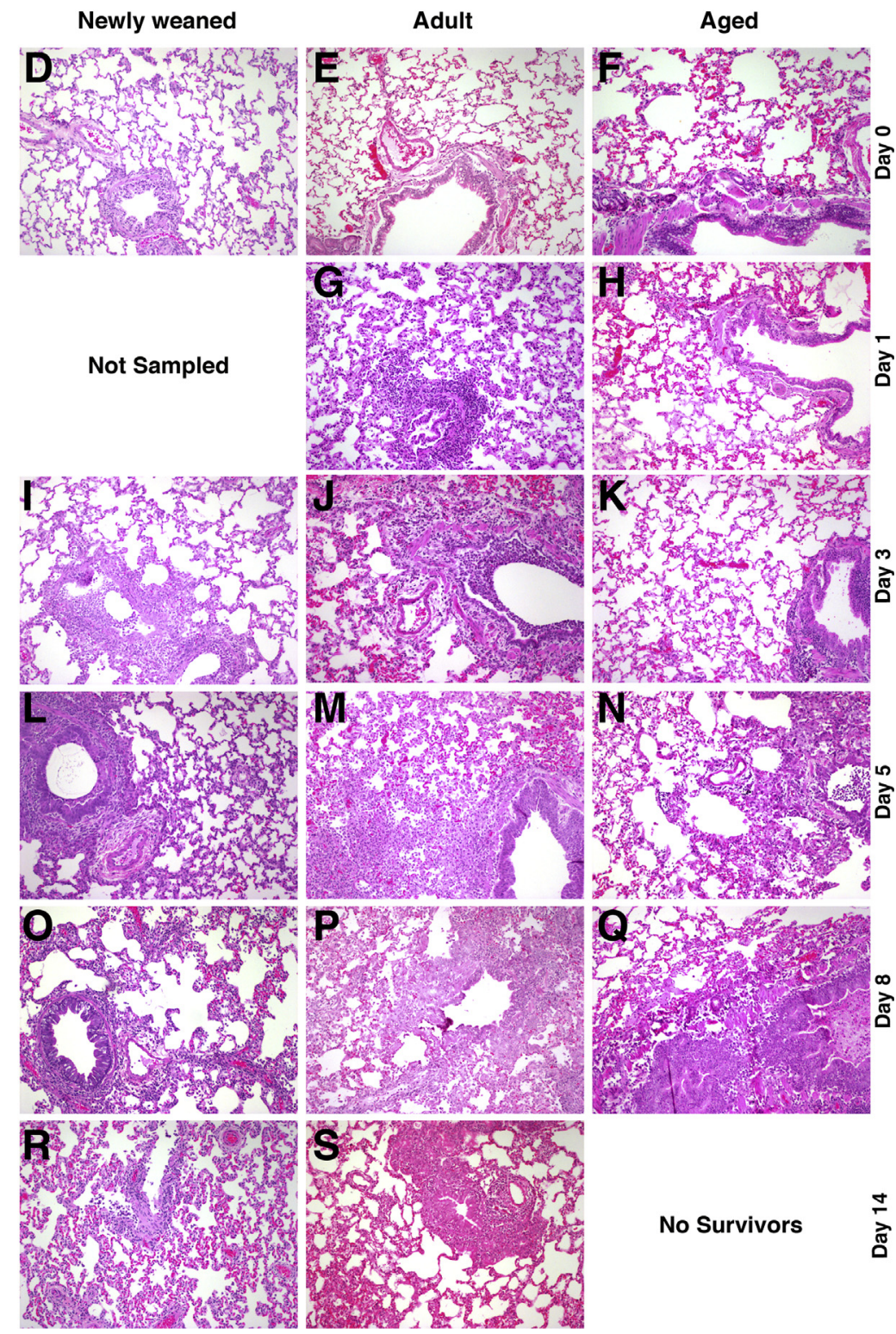

No Survivors
Figure 2 Age-specific development of H1N1 influenza virus pneumonia. A-C: Pneumonia composite scores were assessed for $0,1,3,5,8$, and 14 days post infection for each age group. D-S: Representative images from hematoxylin and eosin-stained sections are depicted for newly weaned $(\mathbf{D}, \mathbf{I}, \mathbf{L}, \mathbf{0}$, and $\mathbf{R})$, adult $(\mathbf{E}, \mathbf{G}, \mathbf{J}, \mathbf{M}, \mathbf{P}$, and $\mathbf{S})$, and aged $(\mathbf{F}, \mathbf{H}$, $\mathbf{K}, \mathbf{N}$, and $\mathbf{Q})$ ferrets during infection $(0,1,3,5,8$, and 14 days post infection). Data are expressed as mean scores (bars) and scores of individual animals (dots) $(\mathbf{A}-\mathbf{C})$. Original magnification, $\times 100($ D-S).
E). Aged ferrets had little bronchial involvement in the early infection period, but severity steadily increased through 8 DPI.

Alveolar Pneumonia Severity Is Strikingly Distinctive in the Age Groups

The presence of alveolar pneumonia differed among the age groups and correlated with survival and weight loss. Newly weaned ferrets had little to no alveolar pneumonia until 8 DPI (Figure 3, C and F), with statistically significant differences compared with adult ferrets at $3(P=0.007$ for lower lobe) and $5(P<0.0001$ for lower lobe) DPI and aged ferrets at $5(P=0.02$ for upper lobe $)$ and $8(P=0.05$ for upper lobe) DPI. By 14 DPI, the mild alveolar pneumonia resolved. Alveolar pneumonia severity was prominent in both adult and aged groups, with adult severity peaking at 5 DPI. As with the bronchial pneumonia, development of 

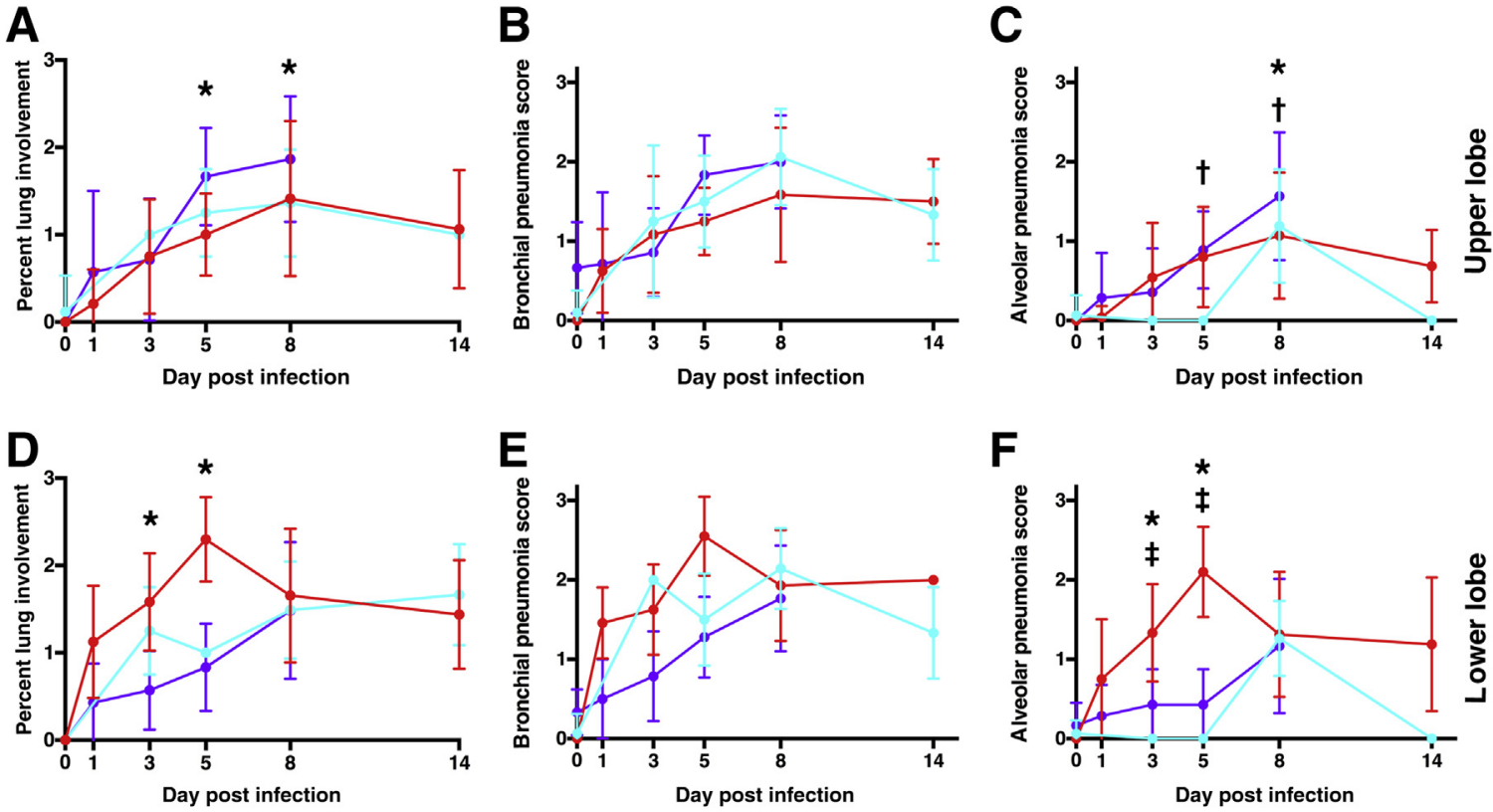

Newly weaned

Adult

Aged

Figure 3 Comparison of global lung involvement and bronchial and alveolar pneumonia severity between each age group. A and $\mathbf{D}$ : The percentage of lung involvement was assessed in lung sections. Lung sections were scored as follows: $0,<10 \% ; 1,10 \%$ to $25 \% ; 2,26 \%$ to $50 \%$; and $3,>50 \%$. B, C, E, and $\mathbf{F}$ : Regional severity of pneumonia was assessed in the bronchi (B and $\mathbf{E})$ and alveolar $(\mathbf{C}$ and $\mathbf{F})$ spaces using the following scoring protocol: 0 , normal; 1 , mild pneumonia; 2, moderate pneumonia; and 3, severe pneumonia. Both the upper $(\mathbf{A}-\mathbf{C})$ and lower $(\mathbf{D}-\mathbf{F})$ left lung lobes were examined. ${ }^{*} P<0.05$ adult versus aged; ${ }^{\dagger} P<0.05$ newly weaned versus aged; ${ }^{\ddagger} P<0.05$ newly weaned versus adult.

alveolar pneumonia in the aged ferrets was delayed and peaked at $8 \mathrm{DPI}$, with statistically significant differences from adults at $3(P=0.004$ for lower lobe $), 5(P<0.0001$ for lower lobe), and 8 ( $P=0.04$ for upper lobe) DPI.

\section{Newly Weaned Ferrets Show Remarkable Protection from Alveolar Cell Infection and Aged Ferrets Are Unable to Clear Infection}

Regional influenza virus infection kinetics also varied among the age groups (Figures 4 and 5). To visualize viral replication, ISH for influenza A virus matrix protein transcripts was performed on sections that contained trachea and left upper and lower lung lobes for each time point (Figure 4). Regional infection severity for trachea, bronchi, submucosal glands, and alveoli were scored. As early as 1 DPI, influenza A virus infection was observed in each region for adult ferrets, whereas aged ferrets had minimal evidence of infection, mostly in the alveolar compartment. Bronchial influenza virus-infected cells peaked in the young ferrets at 3 DPI, in the adult ferrets at 1 to 5 DPI, and at 5 DPI in aged ferrets (Figure 5, A and C). Young and adult ferrets cleared influenza virus infection by $8 \mathrm{DPI}$; however, aged ferrets were unable to clear the virus and had significantly different influenza virus infection compared with adult ferrets at $5(P=0.05$ for upper lobe $)$ and 8 $(P<0.0001$ for upper and lower lobes) DPI and newly weaned ferrets at $3(P=0.008$ for lower lobe $)$ and 8 $(P<0.0001$ for upper and lower lobes) DPI. Although kinetics differed, each age group supported robust bronchial cell infection. Little to no influenza virus was detected in alveolar cells of newly weaned ferrets during the infection (Figure 5, B and D). Adult and aged ferrets had peak alveolar infection at $5 \mathrm{DPI}$, but aged ferrets were unable to clear alveolar infection, with statistically significant differences from adult ferrets at $5(P=0.04$ for upper lobe) and 8 $(P<0.0001$ for upper and lower lobes) DPI and from newly weaned ferrets at $3(P=0.05$ for upper and $P=0.04$ lower lobe), $5(P=0.004$ for upper and lower lobes), and 8 $(P<0.0001$ for upper and lower lobes) DPI. CA/09 influenza $A$ virus infection led to prominent submucosal gland infection in all age groups (Figure 6, A-D), but the kinetics differed, with aged ferrets having statistically significant differences from adult ferrets at $3(P=0.007)$ and 8 $(P<0.0001)$ DPI and newly weaned ferrets at 3 $(P=0.0006)$ and $8(P<0.0001)$ DPI. Trachea infection was most prominent in the newly weaned and aged ferrets, with each cohort having peak viral production at 5 DPI (Figure 6, E-H). Trachea infection was statistically different in aged ferrets compared with adult ferrets at 3 $(P=0.009)$ and $8(P<0.0001)$ DPI and newly weaned ferrets at $3(P=0.0003)$ and $8(P<0.0001)$ DPI.

Pneumonia Is Slow to Develop in Aged Ferrets but Viral Infection Is More Persistent

To visualize a measurement that encompasses the pneumonia severity or the infection severity during influenza 


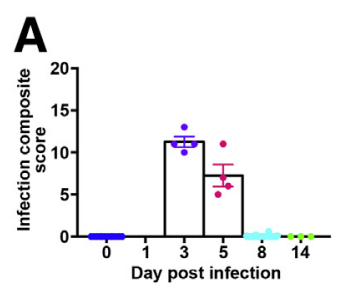

Newly weaned

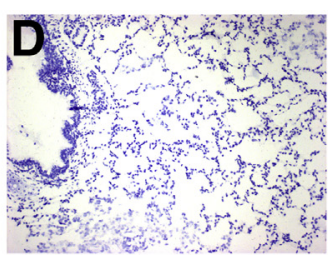

Not Sampled
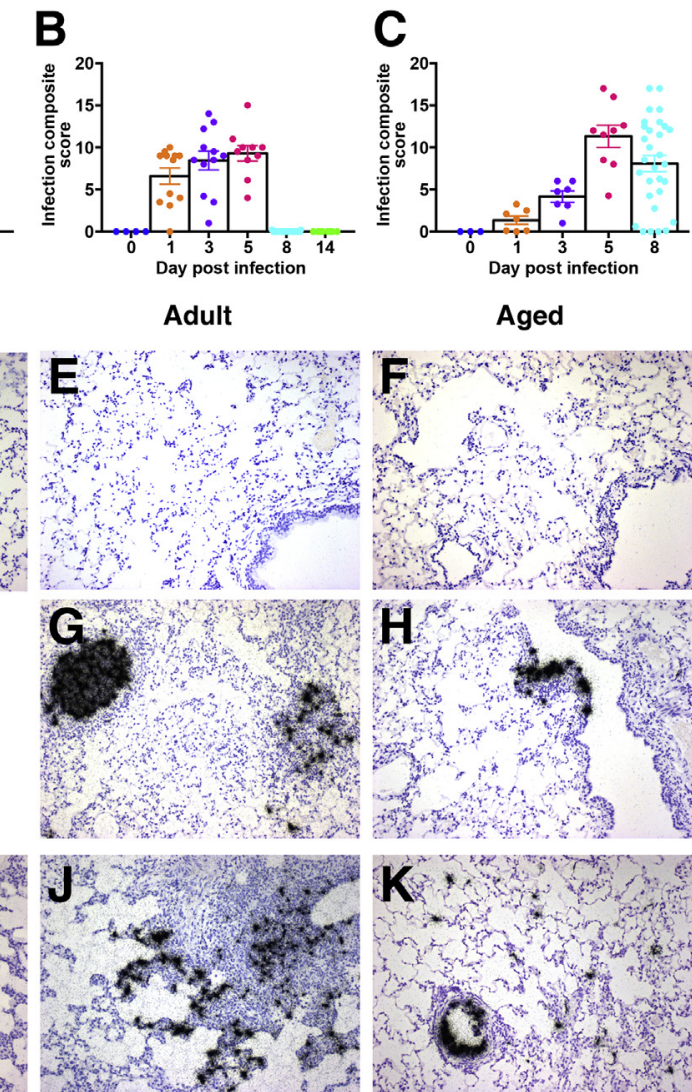

Adult
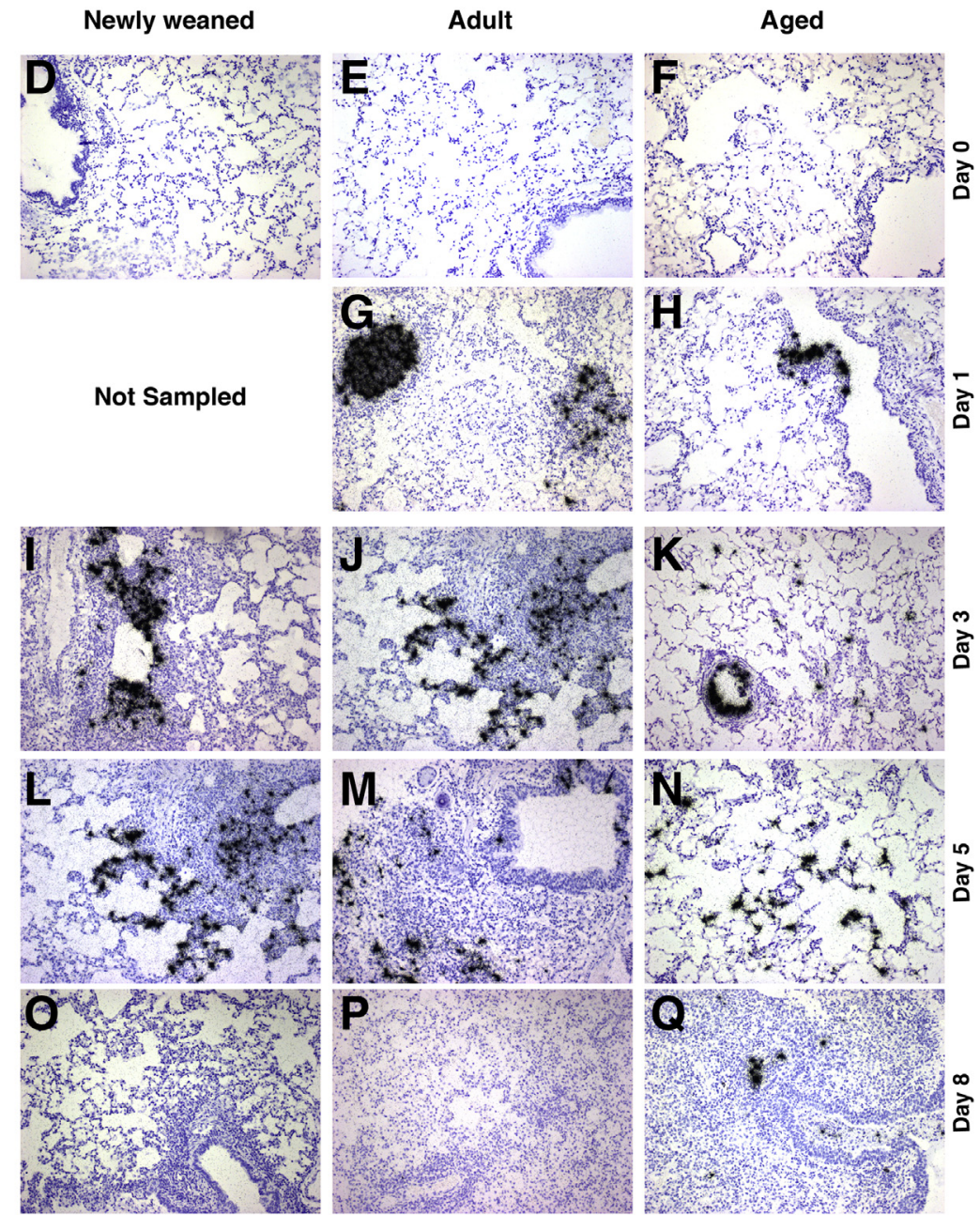

Figure 4 Age-specific H1N1 influenza virus lung infection. A-C: Infection composite scores were assessed for $0,1,3,5,8$, and 14 days post infection for each age group. D-Q: Representative images of lung infection localized by influenza in situ hybridization are depicted for newly weaned (D, I, $\mathbf{L}$, and $\mathbf{0})$, adult $(\mathbf{E}, \mathbf{G}, \mathbf{J}, \mathbf{M}$, and $\mathbf{P})$, and aged $(\mathbf{F}, \mathbf{H}, \mathbf{K}, \mathbf{N}$, and $\mathbf{Q})$ ferrets during infection $(0,1$, 3,5 , and 8 days post infection). Influenza matrix protein RNA transcripts appear as collections of black silver grains over cells counterstained with hematoxylin (blue). Data are expressed as mean scores (bars) and scores of individual animals (dots). Original magnification, $\times 100$ (D-Q). virus infection, a composite score was determined by adding the scores for each category (pneumonia or infection) from the upper and lower lobes at each time point. The pneumonia composite score indicates that pneumonia peaks later in the newly weaned ferrets compared with the mature adults, whereas pneumonia is slow to develop in aged ferrets (Figure 2, A-C). Adult ferrets were slower to resolve signs of pneumonia than newly weaned animals. There was individual ferret variability at most time points for all animals. Despite this variability in aged ferrets at 8 DPI, $100 \%$ of ferrets in this group required euthanasia. The hematoxylin and eosin images of pneumonia during infection reflect the mean composite score shown in Figure 2, A-C (Figure 2, $\mathrm{D}-\mathrm{S}$ ). Infection composite scores have little variability in newly weaned ferrets with peak infection at 3 DPI
(Figure 4). Adult ferrets have greater infection variability throughout the infection phase of the disease and cleared virus by 8 DPI (Figure 4). Aged ferrets also had considerable variability in infection. with a few ferrets having viral clearance by $8 \mathrm{DPI}$, but most aged ferrets had a range of viral infected cells (Figure 4).

\section{Interlobular Variability in Pneumonia and Infection}

Some animals in the adult (33\%) and aged (18\%) groups had marked variation in degree of pneumonia and infection present between the individual upper and lower lung lobes. Infection was more uniform in the newly weaned ferrets. In adults, the greater pathologic findings were usually observed in the lower lung lobe (Supplemental Figure S1, A-F), and 

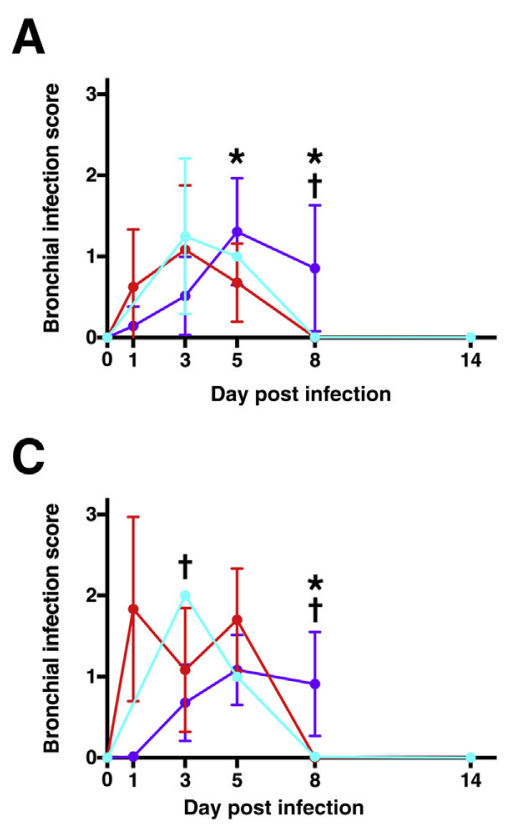

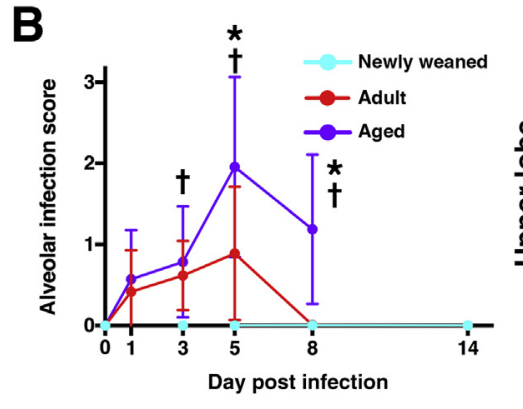

D

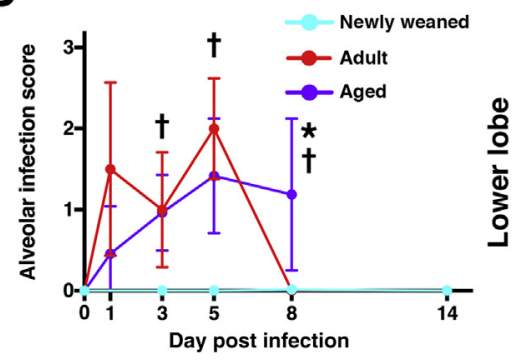

Figure 5 Comparison of regional lung infection severity between each age group. Graphs depict severity of influenza $A$ virus infection in bronchial $(\mathbf{A}$ and $\mathbf{C})$ and alveolar ( $\mathbf{B}$ and $\mathbf{D})$ spaces for the upper ( $\mathbf{A}$ and $\mathbf{B}$ ) and lower (C and $\mathbf{D})$ lung lobes. The severity of influenza infection was determined by scoring of influenza virus in situ hybridization foci: 0 , no definitive signal; 1 , occasional focus; 2 , focus in most fields; and $3,>1$ focus per field. ${ }^{*} P<0.05$ adult versus aged; ${ }^{\dagger} P<0.05$ newly weaned versus aged. examples were found at 1,3 , and 5 DPI. Aged animals did not have disparate involvement until 5 DPI, reflecting the lagging disease (Supplemental Figure S1, G-J).

\section{Discussion}

Influenza virus infection in newly weaned, adult, and aged ferrets produces a range of severities in weight loss, disease symptoms, and virus-induced pathologic findings. Overall, there were age-related differences in the kinetics of CA/09 influenza virus lung infection and the onset and severity of pneumonia. CA/09 influenza virus infected the upper and lower respiratory tracts in all 3 age groups, as reported previously for adult ferrets. ${ }^{33-37}$ In this article, we report that after infection with CA/09 influenza virus, newly weaned ferrets have a relatively mild clinical infection and pneumonia with no alveolar infection, whereas naïve aged ferrets $>5.5$ years old had delayed pneumonia development, less efficient clearance of virus infection, and a higher mortality rate.
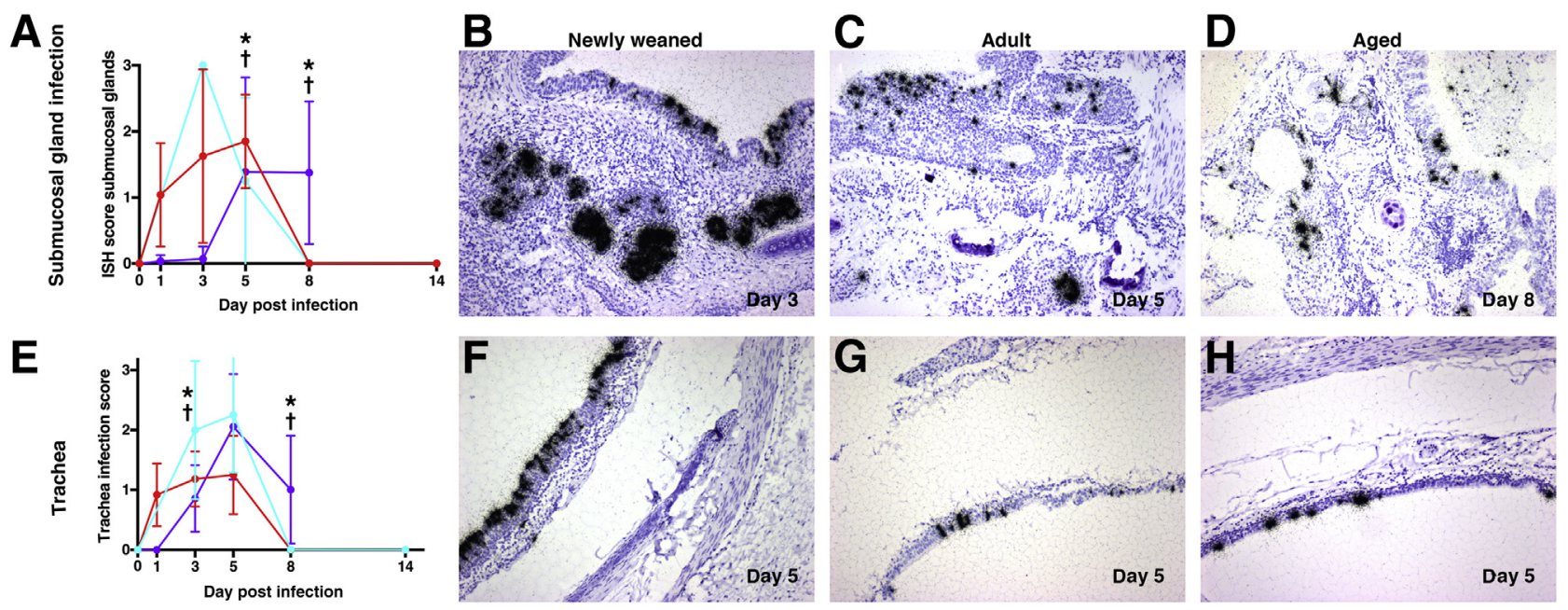

Figure 6 Comparison of submucosal gland $(\mathbf{A}-\mathbf{D})$ or trachea $(\mathbf{E}-\mathbf{H})$ infection severity between each age group. A and E: Submucosal gland $(\mathbf{A})$ or trachea (E) infection severity. Severity of influenza infection was determined by scoring of influenza virus in situ hybridization (ISH) foci: 0 , no definitive signal; 1, occasional focus; 2, focus in most fields; and 3, $>1$ focus per field. B-D: Representative images of submucosal gland infection of the lungs localized by influenza ISH are depicted for newly weaned (B) at 3 days post infection, adult (C) at 5 days post infection, and aged (D) ferrets at 8 days post infection. $\mathbf{F}-\mathbf{H}$ : Representative images of trachea infection localized by influenza ISH are depicted for newly weaned (F), adult $(\mathbf{G})$, and aged $(\mathbf{H})$ ferrets at 5 days post infection. Influenza matrix protein RNA transcripts appear as collections of black silver grains over cells counterstained with hematoxylin (blue). Original magnification, $\times 100$ (B-D and $\mathbf{F}-\mathbf{H}$ ). ${ }^{*} P<0.05$ adult versus aged; ${ }^{\dagger} P<0.05$ newly weaned versus aged. 
Newly weaned ferrets had remarkable resistance to alveolar infection despite abundant tracheal, bronchial, and submucosal gland infection. Infected newly weaned ferrets had a failure to thrive and gain weight compared with noninfected newly weaned ferrets, yet most had less weight loss and pneumonia than the adult and aged ferrets. Severe disease was observed in 9 of 69 infected newly weaned ferrets (13\%) who lost $\geq 15 \%$ body weight by 6 DPI. In contrast, 33 of 63 aged ferrets (52\%) had severe weight loss and disease. In addition to tracheal, bronchial, and submucosal cell infection, adult and aged ferret groups had significant alveolar infection.

Similar to CA/09 influenza virus infection in young children, ${ }^{15-17}$ young ferrets had milder clinical illness than adult and aged ferrets. Compared with adult ferrets, newly weaned ferrets had similar viral clearance kinetics and similar pneumonia profiles but less weight loss and fever. These observations are comparable to previous observations of newly weaned and adult ferrets infected with A/Mexico/ $4108 / 2009$ influenza virus, except that ferrets in the prior study cleared infection earlier and the newly weaned ferrets had earlier resolution of pneumonia. ${ }^{36}$ These newly weaned ferrets also had fewer granulocyte infiltrates in the lung and formation of inducible bronchus-associated lymphoid tissue-like structures. ${ }^{36}$ Inducible bronchus-associated lymphoid tissue is an ectopic lymphoid tissue formed during inflammation or infection and is located throughout the lung. Inducible bronchus-associated lymphoid tissue contributes to pulmonary immune responses by acquiring antigens from the airways, initiating local immune responses, and maintaining lung-resident memory cells. ${ }^{30,38} \mathrm{We}$ hypothesize that these immune mechanisms played an integrated role in reducing severity of influenza virus-induced disease and alveolar cell infection in the newly weaned ferrets.

Adult ferrets had the earliest peak in pneumonia, with a wide range of disease severity. All adult ferrets were able to clear infection by 8 DPI. For this age group, a cohort of male ferrets infected with CA/09 influenza virus was examined to determine sex-related differences in disease severity. Adult female and male ferrets had similar weight loss profiles. Pathologic scores for adult female and male ferrets had a broad, overlapping range of severity in the percentage of lung consolidation and bronchial and alveolar pneumonia with a predominance for bronchial pneumonia (Supplemental Figures S2 and S3). The finding that the severity of pneumonia and lung infection was similar between males and females suggests that sex differences do not overtly affect the parameters examined in this study. We are currently analyzing transcriptomic and genomic data to help determine the mechanisms behind the age group infection severity with a focus on innate immune-related mechanisms.

Interestingly, aged ferrets had a low frequency of infected cells at early time points after infection. Both pneumonia and infection progressed such that by $8 \mathrm{DPI}$, when the other groups had nearly complete viral clearance, aged ferrets still had substantial influenza virus infection in all regional respiratory compartments. A recent study found that aged male ferrets ( $>4$ years of age) sequentially infected with influenza A H1N1 viruses (Bris/59 then Mex/4108) had diminished antibody production and altered peripheral blood $\mathrm{T}$-cell responses compared with adult ferrets (4 to 6 months of age). ${ }^{39}$ Similar to the aged ferrets in this study, aged mice (72 to 76 weeks of age) had delayed responses, increased morbidity, accelerated weight loss, and slower recovery from influenza virus infection compared with adult mice (12 to 16 weeks of age). ${ }^{40}$ Prior exposure of young mice to influenza-like particles that contained hemagglutinin and neuraminidase genes from the 1918 influenza virus at a young age conferred protection from CA/09 virus challenge when they were aged by preventing lower respiratory tract infection, whereas aged control mice succumbed to CA/09 infection by 7 DPI. ${ }^{21}$ This finding suggests that inhibiting lower respiratory tract infection in aged animals limits severity of influenza virus-induced disease.

CA/09-like viruses are more likely to replicate in human and ferret lower respiratory tracts and are more likely to cause pathogenic effects in lung tissue compared with other H1N1 seasonal influenza strains. ${ }^{41-44}$ Lower respiratory tract infection with influenza results in pneumonia and bronchiolitis and is a major cause of morbidity and mortality that disproportionately affects those $>70$ years of age and $<5$ years of age. ${ }^{45}$ When CA/09-like virus induces severe lower respiratory tract infections, it can lead to acute lung injury or acute respiratory failure and carry a high mortality rate. ${ }^{46}$ In this study, ferrets in all age groups had infection of the lower respiratory tract, potentially because of the intranasal infection route. Newly weaned and adult ferrets had comparable bronchial cell infection, yet the newly weaned ferrets had less weight loss. The striking difference in alveolar infection may contribute to the differences in disease outcomes. The CA/09-like strains that emerged and caused the $2009 \mathrm{H} 1 \mathrm{~N} 1$ pandemic resulted in a primarily mild, self-limited illness, requiring hospitalization in $<5 \%$ of infected individuals. However, as observed in the ferrets in this study, there were severe outcomes, including respiratory failure and death. ${ }^{47}$ Both the adult and aged ferret groups had alveolar infection with a wide spectrum of severity, but the aged group had a lethal disease. Thus, the association between location of infection and disease severity attributable to influenza is complicated. Location of inflammation and kinetics of viral replication, viral clearance, and immune response likely factor into disease progression. Examination of immune responses within the local microenvironments of the respiratory tract of each age group would add insight to these dynamics.

Infant ferrets have differences in disease pathogenesis compared with newly weaned ferrets. Infant ferrets infected with CA/09-like viruses have more severe disease and decreased survival compared with adult mothers. ${ }^{48}$ This finding may also recapitulate human disease in which CA/09-like virus-infected infants $<6$ months of age develop 
high rates of hospitalization and death. ${ }^{49-51}$ Influenza viruses that bind to $\alpha_{2-3}$-linked receptors are more likely to infect the lower respiratory tract because the proportion of $\alpha_{2-3}$-linked sialyl glycans is relatively greater than $\alpha_{2-6}$-linked sialyl glycans in both humans and ferrets. ${ }^{52}$ Seasonal H1N1 influenza viruses bind exclusively to $\alpha_{2-6}$-linked sialyl sequences, whereas CA/09-like influenza viruses bind both $\alpha_{2-3}$-linked sialyl and $\alpha_{2-6}$-linked sialyl sequences. ${ }^{53}$ Interrogation of possible differences in expression of sialyl glycans might aid in understanding why newly weaned ferrets are resistant to alveolar cell infection. ${ }^{54}$

This is the first report on the effects of CA/09-like H1N1 influenza viruses in extremely aged ferrets (5.5 to 7 years of age). The naïve aged ferret model appears to resemble humans with severe lower respiratory tract disease and pathologic findings. Despite the delay in lung infection and development of pneumonia, elderly individuals cannot clear influenza virus lung infection as quickly as adults or young people. A range of disease severity was observed with each age group, but there were age-related differences in infection kinetics. Each age group had striking submucosal infection with comparable bronchial pneumonia and infection severity. Newly weaned ferrets had a milder disease than the adult and aged ferrets, with a marked absence of alveolar cell infection. Overall, these ferret models can be used to test vaccines, therapeutics, and drug therapies and to better understand the human immune response to influenza virus infection associated with age.

\section{Acknowledgments}

We thank Mark Stauffer and Kelly Puglisi for valuable technical assistance and preparation of histological samples; and the Influenza Reagent Resource, Influenza Division, World Health Organization Collaborating Center for Surveillance, Epidemiology and Control of Influenza, Centers for Disease Control and Prevention for providing the H1N1 A/California/07/2009 influenza A viruses.

\section{Supplemental Data}

Supplemental material for this article can be found at http://doi.org/10.1016/j.ajpath.2019.08.017.

\section{References}

1. Reed C, Chaves SS, Daily Kirley P, Emerson R, Aragon D, Hancock EB, Butler L, Baumbach J, Hollick G, Bennett NM, Laidler MR, Thomas A, Meltzer MI, Finelli L: Estimating influenza disease burden from population-based surveillance data in the United States. PLoS One 2015, 10:e0118369

2. CDC: Children, the Flu, and the Flu Vaccine. Atlanta, GA, Centers for Disease Control and Prevention, 2017

3. CDC: What You Should Know and Do this Flu Season If You Are 65 Years and Older. Atlanta, GA, Centers for Disease Control and Prevention, 2017
4. Johnson NB, Hayes LD, Brown K, Hoo EC, Ethier KA; Centers for Disease Control and Prevention: CDC National Health Report: leading causes of morbidity and mortality and associated behavioral risk and protective factors-United States, 2005-2013. MMWR Suppl 2014, 63:3-27

5. McElhaney JE, Effros RB: Immunosenescence: what does it mean to health outcomes in older adults? Curr Opin Immunol 2009, 21: 418-424

6. Johnson PL, Yates AJ, Goronzy JJ, Antia R: Peripheral selection rather than thymic involution explains sudden contraction in naive CD4 T-cell diversity with age. Proc Natl Acad Sci U S A 2012, 109: 21432-21437

7. Britanova OV, Putintseva EV, Shugay M, Merzlyak EM, Turchaninova MA, Staroverov DB, Bolotin DA, Lukyanov S, Bogdanova EA, Mamedov IZ, Lebedev YB, Chudakov DM: Agerelated decrease in TCR repertoire diversity measured with deep and normalized sequence profiling. J Immunol 2014, 192:2689-2698

8. Montecino-Rodriguez E, Berent-Maoz B, Dorshkind K: Causes, consequences, and reversal of immune system aging. J Clin Invest 2013, 123:958-965

9. Nikolich-Zugich J: The twilight of immunity: emerging concepts in aging of the immune system. Nat Immunol 2018, 19:10-19

10. Kang I, Hong MS, Nolasco H, Park SH, Dan JM, Choi JY, Craft J: Age-associated change in the frequency of memory CD4 $+\mathrm{T}$ cells impairs long term CD4 $+\mathrm{T}$ cell responses to influenza vaccine. J Immunol 2004, 173:673-681A

11. Chowell G, Bertozzi SM, Colchero MA, Lopez-Gatell H, AlpucheAranda C, Hernandez M, Miller MA: Severe respiratory disease concurrent with the circulation of H1N1 influenza. N Engl J Med 2009, 361:674-679

12. Ede LC, Loeffelholz MJ, Alvarez-Fernandez P, Pong DL, Patel JA, McCormick DP, Chonmaitree T: Effect of the 2009 influenza $\mathrm{A} / \mathrm{H} 1 \mathrm{~N} 1$ pandemic on viral respiratory infections in the first year of life. Pediatr Infect Dis J 2012, 31:1107-1112

13. Finelli L, Fiore A, Dhara R, Brammer L, Shay DK, Kamimoto L, Fry A, Hageman J, Gorwitz R, Bresee J, Uyeki T: Influenza-associated pediatric mortality in the United States: increase of Staphylococcus aureus coinfection. Pediatrics 2008, 122:805-811

14. Cox CM, Blanton L, Dhara R, Brammer L, Finelli L: 2009 Pandemic influenza A (H1N1) deaths among children-United States, 20092010. Clin Infect Dis 2011, 52 Suppl 1:S69-74

15. Helferty M, Vachon J, Tarasuk J, Rodin R, Spika J, Pelletier L: Incidence of hospital admissions and severe outcomes during the first and second waves of pandemic (H1N1) 2009. CMAJ 2010, 182: $1981-1987$

16. Martic J, Savic N, Minic P, Pasic S, Nedeljkovic J, Jankovic B: Novel H1N1 influenza in neonates: from mild to fatal disease. J Perinatol 2011, 31:446-448

17. Halasa NB: Update on the 2009 pandemic influenza A H1N1 in children. Curr Opin Pediatr 2010, 22:83-87

18. Verma N, Dimitrova M, Carter DM, Crevar CJ, Ross TM, Golding H, Khurana S: Influenza virus H1N1pdm09 infections in the young and old: evidence of greater antibody diversity and affinity for the hemagglutinin globular head domain (HA1 domain) in the elderly than in young adults and children. J Virol 2012, 86:5515-5522

19. Hancock K, Veguilla V, Lu X, Zhong W, Butler EN, Sun H, Liu F Dong L, DeVos JR, Gargiullo PM, Brammer TL, Cox NJ, Tumpey TM, Katz JM: Cross-reactive antibody responses to the 2009 pandemic H1N1 influenza virus. N Engl J Med 2009, 361: $1945-1952$

20. Xie H, Li X, Gao J, Lin Z, Jing X, Plant E, Zoueva O, Eichelberger MC, Ye Z: Revisiting the 1976 "swine flu" vaccine clinical trials: cross-reactive hemagglutinin and neuraminidase antibodies and their role in protection against the $2009 \mathrm{H} 1 \mathrm{~N} 1$ pandemic virus in mice. Clin Infect Dis 2011, 53:1179-1187

21. Giles BM, Bissel SJ, Craigo JK, Dealmeida DR, Wiley CA, Tumpey TM, Ross TM: Elicitation of anti-1918 influenza virus 
immunity early in life prevents morbidity and lower levels of lung infection by 2009 pandemic H1N1 influenza virus in aged mice. J Virol 2012, 86:1500-1513

22. Xu R, Ekiert DC, Krause JC, Hai R, Crowe JE Jr, Wilson IA: Structural basis of preexisting immunity to the 2009 H1N1 pandemic influenza virus. Science 2010, 328:357-360

23. Carter DM, Darby CA, Johnson SK, Carlock MA, Kirchenbaum GA, Allen JD, Vogel TU, Delagrave S, DiNapoli J, Kleanthous H, Ross TM: Elicitation of protective antibodies against a broad panel of H1N1 viruses in ferrets preimmune to historical H1N1 influenza viruses. J Virol 2017, 91

24. Belser JA, Katz JM, Tumpey TM: The ferret as a model organism to study influenza A virus infection. Dis Model Mech 2011, 4:575-579

25. Enkirch T, von Messling V: Ferret models of viral pathogenesis. Virology 2015, 479-480:259-270

26. Committee for the Update of the Guide for the Care and Use of Laboratory AnimalsNational Research Council: Guide for the Care and Use of Laboratory Animals: Eighth Edition. Washington, DC, National Academies Press, 2011

27. Tobita K: Permanent canine kidney (MDCK) cells for isolation and plaque assay of influenza B viruses. Med Microbiol Immunol 1975, $162: 23-27$

28. Tobita K, Sugiura A, Enomote C, Furuyama M: Plaque assay and primary isolation of influenza A viruses in an established line of canine kidney cells (MDCK) in the presence of trypsin. Med Microbiol Immunol 1975, 162:9-14

29. Giles BM, Bissel SJ, Dealmeida DR, Wiley CA, Ross TM: Antibody breadth and protective efficacy are increased by vaccination with computationally optimized hemagglutinin but not with polyvalent hemagglutinin-based $\mathrm{H} 5 \mathrm{~N} 1$ virus-like particle vaccines. Clin Vaccine Immunol 2012, 19:128-139

30. Hwang JY, Randall TD, Silva-Sanchez A: Inducible bronchusassociated lymphoid tissue: taming inflammation in the lung. Front Immunol 2016, 7:258

31. Bissel SJ, Giles BM, Wang G, Olevian DC, Ross TM, Wiley CA: Acute murine H5N1 influenza A encephalitis. Brain Pathol 2012, 22: $150-158$

32. Huang SS, Banner D, Fang Y, Ng DC, Kanagasabai T, Kelvin DJ, Kelvin AA: Comparative analyses of pandemic H1N1 and seasonal H1N1, H3N2, and influenza B infections depict distinct clinical pictures in ferrets. PLoS One 2011, 6:e27512

33. Rowe T, Leon AJ, Crevar CJ, Carter DM, Xu L, Ran L, Fang Y, Cameron CM, Cameron MJ, Banner D, Ng DC, Ran R, Weirback HK, Wiley CA, Kelvin DJ, Ross TM: Modeling host responses in ferrets during A/California/07/2009 influenza infection. Virology 2010, 401:257-265

34. Carolan LA, Rockman S, Borg K, Guarnaccia T, Reading P, Mosse J, Kelso A, Barr I, Laurie KL: Characterization of the localized immune response in the respiratory tract of ferrets following infection with influenza A and B viruses. J Virol 2016, 90:2838-2848

35. Maines TR, Jayaraman A, Belser JA, Wadford DA, Pappas C, Zeng H, Gustin KM, Pearce MB, Viswanathan K, Shriver ZH, Raman R, Cox NJ, Sasisekharan R, Katz JM, Tumpey TM: Transmission and pathogenesis of swine-origin $2009 \mathrm{~A}(\mathrm{H} 1 \mathrm{~N} 1)$ influenza viruses in ferrets and mice. Science 2009, 5939:484-487

36. Huang SS, Banner D, Degousee N, Leon AJ, Xu L, Paquette SG, Kanagasabai T, Fang Y, Rubino S, Rubin B, Kelvin DJ, Kelvin AA: Differential pathological and immune responses in newly weaned ferrets are associated with a mild clinical outcome of pandemic 2009 H1N1 infection. J Virol 2012, 86:13187-13201

37. Iyer SS, Cheng G: Role of interleukin 10 transcriptional regulation in inflammation and autoimmune disease. Crit Rev Immunol 2012, 32:23-63

38. Randall TD: Bronchus-associated lymphoid tissue (BALT) structure and function. Adv Immunol 2010, 107:187-241

39. Paquette SG, Huang SSH, Banner D, Xu L, Leomicronn A, Kelvin AA, Kelvin DJ: Impaired heterologous immunity in aged ferrets during sequential influenza A H1N1 infection. Virology 2014, 464-465:177-183
40. Toapanta FR, Ross TM: Impaired immune responses in the lungs of aged mice following influenza infection. Respir Res 2009, 10:112

41. van den Brand JM, Stittelaar KJ, van Amerongen G, Rimmelzwaan GF, Simon J, de Wit E, Munster V, Bestebroer T, Fouchier RA, Kuiken T, Osterhaus AD: Severity of pneumonia due to new H1N1 influenza virus in ferrets is intermediate between that due to seasonal H1N1 virus and highly pathogenic avian influenza H5N1 virus. J Infect Dis 2010, 201:993-999

42. Cheng VC, To KK, Tse H, Hung IF, Yuen KY: Two years after pandemic influenza A/2009/H1N1: what have we learned? Clin Microbiol Rev 2012, 25:223-263

43. Safronetz D, Rockx B, Feldmann F, Belisle SE, Palermo RE, Brining D, Gardner D, Proll SC, Marzi A, Tsuda Y, Lacasse RA, Kercher L, York A, Korth MJ, Long D, Rosenke R, Shupert WL, Aranda CA, Mattoon JS, Kobasa D, Kobinger G, Li Y, Taubenberger JK, Richt JA, Parnell M, Ebihara H, Kawaoka Y, Katze MG, Feldmann H: Pandemic swine-origin H1N1 influenza A virus isolates show heterogeneous virulence in macaques. J Virol 2011, 85:1214-1223

44. Camp JV, Bagci U, Chu YK, Squier B, Fraig M, Uriarte SM, Guo H, Mollura DJ, Jonsson CB: Lower respiratory tract infection of the ferret by 2009 H1N1 pandemic influenza A virus triggers biphasic, systemic, and local recruitment of neutrophils. J Virol 2015, 89: 8733-8748

45. GBD 2015 LRI Collaborators: Estimates of the global, regional, and national morbidity, mortality, and aetiologies of lower respiratory tract infections in 195 countries: a systematic analysis for the Global Burden of Disease Study 2015. Lancet Infect Dis 2017, 17: $1133-1161$

46. Kumar TC, Shivakumar NS, Deepak TS, Krishnappa R, Goutam MS, Ganigar V: H1N1-infected patients in ICU and their clinical outcome. N Am J Med Sci 2012, 4:394-398

47. Dawood FS, Jain S, Finelli L, Shaw MW, Lindstrom S, Garten RJ, Gubareva LV, Xu X, Bridges CB, Uyeki TM; Novel Swine-Origin Influenza A (H1N1) Virus Investigation Team: Emergence of a novel swine-origin influenza A (H1N1) virus in humans. N Engl J Med 2009, 360:2605-2615

48. Paquette SG, Banner D, Huang SS, Almansa R, Leon A, Xu L, Bartoszko J, Kelvin DJ, Kelvin AA: Influenza transmission in the mother-infant dyad leads to severe disease, mammary gland infection, and pathogenesis by regulating host responses. PLoS Pathog 2015, $11: \mathrm{e} 1005173$

49. Van Kerkhove MD, Vandemaele KA, Shinde V, JaramilloGutierrez G, Koukounari A, WHO Working Group for Risk Factors for Severe H1N1pdm Infection, et al: Risk factors for severe outcomes following 2009 influenza A (H1N1) infection: a global pooled analysis. PLoS Med 2011, 8:e1001053

50. Louie JK, Gavali S, Acosta M, Samuel MC, Winter K, Jean C, Glaser CA, Matyas BT, Schechter R; California Pandemic (H1N1) Working Group: Children hospitalized with 2009 novel influenza A(H1N1) in California. Arch Pediatr Adolesc Med 2010, 164: $1023-1031$

51. Libster R, Bugna J, Coviello S, Hijano DR, Dunaiewsky M, Reynoso N, et al: Pediatric hospitalizations associated with 2009 pandemic influenza A (H1N1) in Argentina. N Engl J Med 2010, 362 : $45-55$

52. Shinya K, Ebina M, Yamada S, Ono M, Kasai N, Kawaoka Y: Avian flu: influenza virus receptors in the human airway. Nature 2006, 440: 435-436

53. Childs RA, Palma AS, Wharton S, Matrosovich T, Liu Y, Chai W, Campanero-Rhodes MA, Zhang Y, Eickmann M, Kiso M, Hay A, Matrosovich M, Feizi T: Receptor-binding specificity of pandemic influenza A (H1N1) 2009 virus determined by carbohydrate microarray. Nat Biotechnol 2009, 27:797-799

54. Cerna A, Janega P, Martanovic P, Lisy M, Babal P: Changes in sialic acid expression in the lung during intrauterine development of the human fetus. Acta Histochem 2002, 104:339-342 